MATHEMATICAL ASSOCIATION

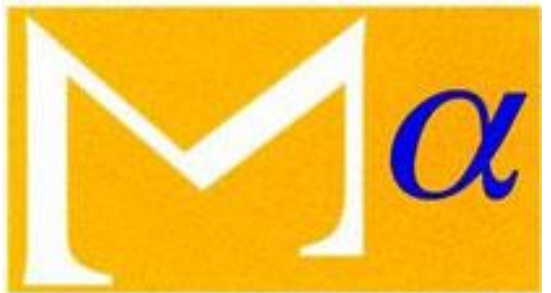

supporting mathematics in education

The Introduction to Infinite Series

Author(s): W. J. Dobbs

Source: The Mathematical Gazette, Vol. 9, No. 135 (May, 1918), pp. 242-246

Published by: Mathematical Association

Stable URL: http://www.jstor.org/stable/3605310

Accessed: 21-12-2015 05:52 UTC

Your use of the JSTOR archive indicates your acceptance of the Terms \& Conditions of Use, available at http://www.jstor.org/page/ info/about/policies/terms.jsp

JSTOR is a not-for-profit service that helps scholars, researchers, and students discover, use, and build upon a wide range of content in a trusted digital archive. We use information technology and tools to increase productivity and facilitate new forms of scholarship. For more information about JSTOR, please contact support@jstor.org. 


\title{
THE INTRODUCTION TO INFINITE SERIES.*
}

\author{
Br W. J. Dobes, M.A.
}

IN the Report of the Education Reform Council, published last year under the title "Education Reform," a paragraph occurs on page 84 with reference to the Mathematics Course suitable for boys of 16 to 18 years of age attending a Secondary School. There it is suggested that such boys who take up Mathematics and Science may pursue the study of differential and integral calculus, including the easy parts of differential equations, and apply these with advantage to higher algebra, trigonometry, coordinate geometry, solid geometry and mechanics; that the course should include some introduction to infinite series; that too much time has been devoted hitherto to the binomial series; and that the thing to aim at is Taylor's series.

The school course in Higher Mathematics with which most of us are familiar involves much work of little educational value on the binomial series, including the extension from a positive integral index to other indices, i.e. from a finite to an intinite series, as given, for instance, in Todhunter's Algebra. Then the exponential, logarithmic, and trigonometrical series are based upon the binomial series. Finally, a very few schoolboys are introduced to Taylor's series by assuming the existence and validity of an expansion in ascending integral powers of the variable. Any treatment of infinite series suitable for the ordinary schoolboy is hampered by the difficulties inseparable from considerations of convergency. Yet it seems desirable that schoolboys should have some acquaintance with the most important of these series and be able to apply them to practical use. The problem is an important one, but a very thorny one, and I hope that some mathematician familiar with the limitations of the schoolroom, but more able to deal with it than I can claim to be, will take the matter up. The treatment should be such that the boy will have nothing to unlearn, and that he may acquire such knowledge as he can put to practical use. It is not necessary that the course should be completely rigorous from beginning to end, but it is desirable that all the steps should be capable of a rigorous justification perhaps at a later stage. It may not infrequently happen that an assumption is made which the boy may not at the time be able to justify; but such assumptions should be honestly faced and admitted. And we must never argue about an infinite number of quantities as we would about a finite number.

In a recently published school algebra t by a well-known writer who formerly thought differently, the binomial, exponential and logarithmic infinite series are now merely stated without proof, and their uses illustrated. Perhaps this will turn ont to be the only practicable method for school work, but I venture to hope that a better method may be found. I wish to place before this association a few suggestions for consideration. If they do not stand the test of searching criticism, they may perhaps stimulate others to attack the problem with greater success.

The early introduction of calculus methods into school work, if developed along suitable lines, provides a quicker, and perhaps a better, means of access to these series than was possible when the vast majority of schoolboys were denied access to the elements of infinitesimal calculus. Clearly, if the calculus method is to be available, the rules for differentiating the standard functions must not be based on the accepted expansions of those functions in infinite series.

* Read at a meeting of the London branch on the 9th of March, 1918. + $A$ School Algebra, by H. S. Hall. 
The preliminary work also involves a discussion of the properties of the function $y=a^{x}$, where $a$ is a positive quantity. This arises in connection with the theory of indices and logarithms. The addition of $\frac{1}{n}$ to the value of $x$ applies the growth-factor $\sqrt[n]{ } a$ to the value of $y, n$ being a positive integer. If $a>1, y$ increases without limit as $x \rightarrow \infty$, and $y$, remaining positive, can be made $<\epsilon$ by sufficiently increasing numerically the negative value of $x, \epsilon$ denoting a quantity to which any given positive value may be assigned as small as we please. If $a<1, y$, remaining positive, can be made $<\epsilon$ by sufficiently increasing the positive value of $x$, and $y$ increases without limit as $x \rightarrow-\infty$. The gradient of the graph is proportional to $y$ and becomes identical with $y$ when $a$ has the special value $e$. This association has already suffered at my hands a paper on this subject a few years ago.*

Let us suppose then that, instead of spending much time upon the binomial series and other more or less undesirable topics, the schoolboy has learned how to differentiate such functions as $x^{a}, a^{x}, \log x, \sin x, \cos x, \tan ^{-1} x$. Also, in connection with the consideration of irrational numbers, he has been introduced to the idea of a sequence. Recently Professor Carey's Infinitesimal Calculus has presented this topic in a very attractive form, and I heartily commend his book to your notice.

We will consider in turn a few of the most important infinite series. I shall throughout use the symbol $n$ to denote a positive integer and the symbol $\epsilon$ to denote a quantity to which any given positive value may be assigned as small as we please.

(i) Expansions of $\sin x$ and $\cos x$. We will take as our first examples the expansions of $\sin x$ and $\cos x$ in ascending powers of $x$. The schoolboy requires these expansions only for values of $x$ ranging from 0 to $\frac{1}{4} \pi$, i.e. for positive angles less than $45^{\circ}$. We will, however, limit ourselves to values of $x$ ranging between 0 and 1, i.e. to angles ranging between 0 and about $57 \cdot 3^{\circ}$.
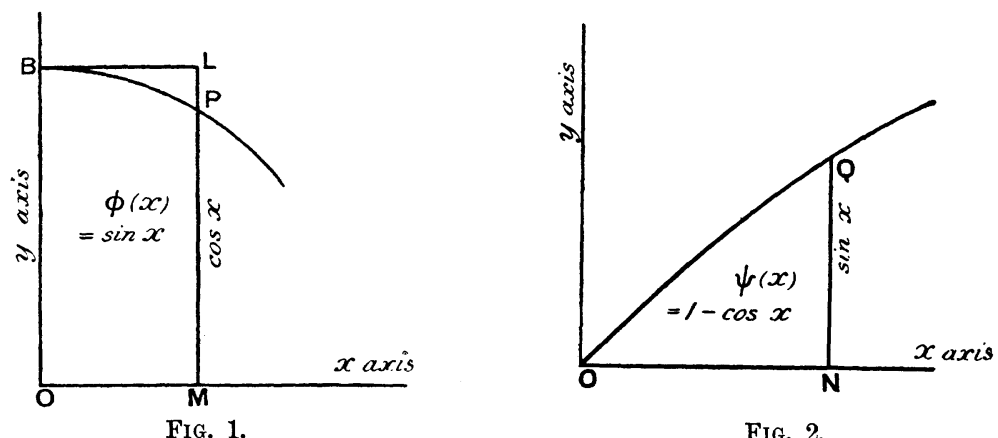

FIG. 2 .

In the left-hand figure, which represents the graph of $y=\cos x$ for values of $x$ ranging between 0 and 1 , let $\phi(x)$ denote the area $O M P B$ between the axes of coordinates, any ordinate $M P$ and the curve. In the right-hand figure, which represents the graph of $y=\sin x$ for values of $x$ ranging between 0 and 1 , let $\psi(x)$ denote the area $O N Q$ between the $x$ axis, any ordinate $N Q$ and the curve.

Then

Also

$$
\begin{gathered}
\phi^{\prime}(x)=\cos x \text { and } \phi(0)=0 ; \\
\therefore \phi(x)=\sin x . \\
\psi^{\prime}(x)=\sin x \text { and } \psi(0)=0 ; \\
\therefore \psi(x)=1-\cos x .
\end{gathered}
$$

* See Mathematical Gazette, vol. v. p. 179. 
Now, except when $x=0, \cos x<1$;

$$
\begin{gathered}
\therefore \text { area } O M P B<\text { area } O M L B, \\
\text { i.e. } \sin x<x .
\end{gathered}
$$

Thus

$$
\begin{aligned}
\sin x>0 & \text { but }<x ; \\
\therefore \psi(x)>0 & \text { but }<\frac{1}{2} x^{2},
\end{aligned}
$$

i.e. $1-\cos x>0$ but $<\frac{1}{2} x^{2}$;

$\therefore \cos x<1$ but $>1-\frac{1}{2} x^{2}$;

$\therefore \phi(x)<x$ but $>x-\frac{x^{3}}{3}$,

i.e. $\sin x<x$ but $>x-\frac{x^{3}}{3}$

$\therefore \psi(x)<\frac{1}{2} x^{2}$ but $>\frac{x^{2}}{\underline{2}}-\frac{x^{4}}{\mid \underline{4}}$,

i.e. $1-\cos x<\frac{x^{2}}{\underline{L}}$ but $>\frac{x^{2}}{2}-\frac{x^{4}}{\square}$;

$\therefore \cos x>1-\frac{x^{2}}{\sqrt{2}}$ but $<1-\frac{x^{2}}{\sqrt{2}}+\frac{x^{4}}{\sqrt{4}}$;

$\therefore \phi(x)>x-\frac{x^{3}}{\mid 3}$ but $<x-\frac{x^{3}}{\mid 3}+\frac{x^{5}}{\square}$,

i.e. $\sin x>x-\frac{\overline{x^{3}}}{\underline{3}}$ but $<x-\frac{x^{3}}{\underline{3}}+\frac{x^{5}}{\underline{5}}$,

and so onl.

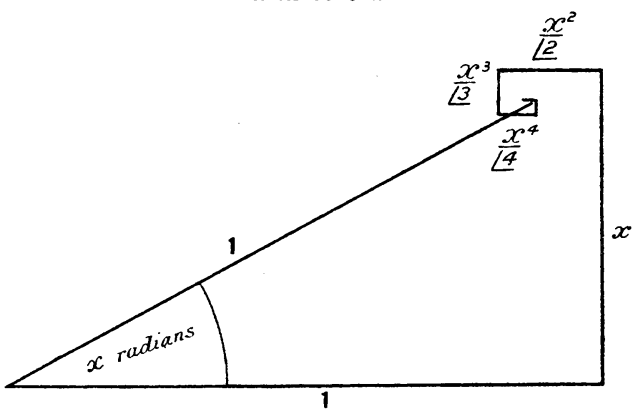

FIG. 3.

Thus we have the sequences of functions

$$
\left\{\begin{array}{l}
1, \\
1-\frac{x^{2}}{\frac{2}{x^{2}}}, \\
1-\frac{x^{2}}{\frac{2}{\mid 2}}+\frac{x^{4}}{\mid 4}, \\
\text { etc. }
\end{array}\right\} \text { and }\left\{\begin{array}{l}
x, \\
x-\frac{x^{3}}{\sqrt{3}}, \\
x-\frac{x^{3}}{\sqrt{3}}+\frac{x^{5}}{\mid 5}, \\
\text { etc. }
\end{array}\right\}
$$

the former giving successive approximations to $\cos x$ alternately too great and too small, the latter giving successive approximations to $\sin x$ alternately too great and too small. In both series every new term introduced is smaller than that which preceded it, and each term is of the form $\pm \frac{x^{n}}{[n}$, the 
numerical value of which can be made $<\epsilon$ by sufficiently increasing $n$. Here we have two ideal series for purposes of calculation, and can at every stage assign an outside estimate to the error remaining after a finite number of terms has been used. Though $x$ may be limited to values ranging from 0 to $\frac{1}{4} \pi$, we have here suitable machinery for the calculation, to any required degree of accuracy, of any trigonometrical function of any angle. These expansions are therefore particularly suitable for school use.* The figure on p. 244 is an obvious geometrical illustration.

(ii) Geometric Progression. Secondly, let us take the familiar series whose general term, the $(n+1)$ th, is $x^{n}$, and, denoting $\frac{1}{1-x}$ by $f$, consider the sequence

$$
\begin{aligned}
& \phi_{1}=1+x=f-x^{2} f, \\
& \phi_{2}=1+x+x^{2}=f-x^{3} f \text {, } \\
& \phi_{n}=1+x+x^{2}+\ldots+x^{n}=f-x^{n+1} f .
\end{aligned}
$$

If $x$ is numerically less than $p$, where $p$ is a positive quantity less than 1 , $x^{n}$ can be made numerically $<\epsilon$ by sufficiently increasing $n$, and $f$ remains finite, though it varies with $x$ between the limits $\frac{1}{1+p}$ and $\frac{1}{1-p}$. Thus the limit of the sequence is $f$ for all values of $x$ ranging from $-p$ to $+p$. In writing $f$ as an approximation to the sum of a finite number $n$ of terms of the series, we know that the error is $x^{n} f$ exactly.

A very beautiful and illuminating geometrical illustration of this convergence is given in Carslaw's Plane Trigonometry. Take a line-segment $O A$ of unit length; and draw $O P$ of gradient $x$ and $A P$ of gradient 1 , meeting at

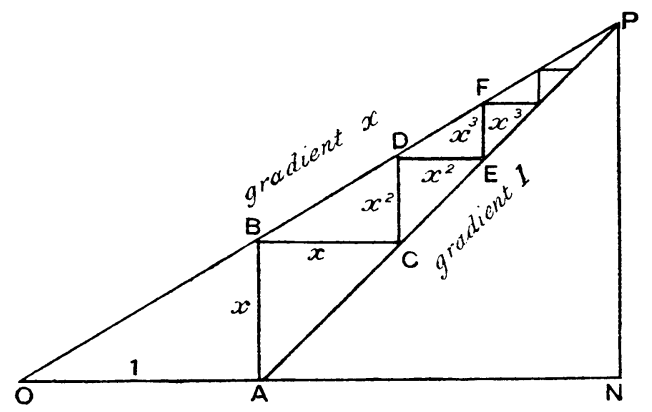

FIG. 4.

$P$. Then $O A, A B, B C, C D, D E, E F^{\prime}$, etc., drawn successively at right angles, have the values $1, x, x, x^{2}, x^{2}, x^{3}$, etc. Draw $P N$ perpendicular upon $O A$ produced. Then $O N$ is the limit of the sum $1+x+x^{2}+\ldots$, and denoting this by $s, N P=s-1$. Clearly $s-1=x s$; whence $s=\frac{1}{1-x}=f$. The remainder $x^{n} f$ may also be obtained directly from the figure.

(iii) Logarithmic Series. Knowing that

$$
\frac{d \log (1+x)}{d x}=\frac{1}{1+x}
$$

* On this point Professor Nunn has anticipated me. Since this paper was written I have found the expansions of the sine and cosine treated in the same way on pages 308 and 309 of his Exercises in Algebra, Part II. 
the preceding expansion suggests that, provided $x$ is numerically less than 1 , we may, with adequate reservations, write

$$
\frac{d \log (1+x)}{d x}=1-x+x^{2}+\ldots
$$

May we integrate, and, omitting the constant of integration because $\log (1+x)=0$ when $x=0$, say

$$
\log (1+x)=x-\frac{1}{2} x^{2}+\frac{1}{3} x^{3}-\ldots ?
$$

This step requires justification and elucidation.

Consider the sequence of functions

$$
\begin{aligned}
& \phi_{1}=\log (1+x)-x \text {, } \\
& \phi_{2}=\log (1+x)-x+\frac{1}{2} x^{2} \text {, } \\
& \phi_{3}=\log (1+x)-x+\frac{1}{2} x^{2}-\frac{1}{3} x^{3}, \\
& \phi_{n}=\log (1+x)-x+\frac{1}{2} x^{2}-\ldots+(-1)^{n} \frac{1}{n} x^{n} .
\end{aligned}
$$

We notice that these functions have a common value 0 when $x$ is 0 .

Denoting differentiation with respect to $x$ by a dash, we have

$$
\begin{aligned}
\phi_{n}{ }^{\prime} & =\frac{1}{1+x}-1+x-x^{2}+\ldots+(-1)^{n} x^{n-1} \\
& =(-1)^{n} x^{n}\left(\frac{1}{1+x}\right), \quad \text { summing the G.P. }
\end{aligned}
$$

Thus, corresponding to the sequence of $\phi$ 's, we have the following sequence of functions expressing the gradient-laws of the former sequence :

$$
\begin{aligned}
& \phi_{1}{ }^{\prime}=-x\left(\frac{1}{1+x}\right), \\
& \phi_{2}{ }^{\prime}=+x^{2}\left(\frac{1}{1+x}\right), \\
& \phi_{3}{ }^{\prime}=-x^{3}\left(\frac{1}{1+x}\right), \\
& \cdots \ldots \ldots \ldots \ldots \ldots \ldots \ldots \ldots \ldots \ldots \ldots \ldots \ldots \\
& \phi_{n}{ }^{\prime}=(-1)^{n} x^{n}\left(\frac{1}{1+x}\right) .
\end{aligned}
$$

We notice that each of these functions is 0 when $x$ is 0 .

Now, when $x$ ranges in value between $-p$ and $+p$, where $p$ is a positive quantity less than 1 , the numerical value of $x^{n}$ can always be made as small as we please by sufficiently increasing $n$; at the same time $\frac{1}{1+x}$ remains finite, though it varies with $x$ between the limits $\frac{1}{1-p}$ and $\frac{1}{1+p}$. Hence, for all values of $x$ ranging from $-p$ to $+p, \phi_{n}{ }^{\prime}$ can be made numerically less than $\epsilon$ by sufficiently increasing $n$. For obvious reasons I have avoided values of $x$ in the immediate neighbourhood of \pm 1 . Indeed, for school purposes, it would be sufficient to allow $x$ to range from $-\frac{1}{2}$ and $+\frac{1}{2}$.

Now, the graphs of $\phi_{1}, \phi_{2}, \phi_{3}, \ldots$ all touch the $x$ axis at the origin, and their gradients all $\rightarrow 0$ throughout the range $-p$ to $+p$ of $x$ when $n \rightarrow \infty$. Thus it appears that for values of $x$ ranging from $-p$ to $+p$ all the graphs tend towards the $x$ axis as the limit when $n \rightarrow \infty$. May we infer that within this range $\phi_{n} \rightarrow 0$ when $n \rightarrow \infty$, i.e. that $\log (1+x)$ is the limit of the sum $x-\frac{1}{2} x^{2}+\frac{1}{3} x^{3}-\ldots$ when the number of terms is indefinitely increased? We will consider this point a little more closely ; and, as a similar digression will be required in connection with other series, we will take a more general case than the present one.

( $T$ o be continued.) 